\title{
Construcción de subjetividad trans a partir de la ocupación entendida como hacer, ser y llegar a ser
}

\author{
Construção da subjetividade trans a partir da ocupação compreendida \\ como fazer, ser e chegar a ser \\ Construction of trans subjectivity from the occupation understood as doing, \\ being and becoming
}

Bárbara Francisca Gómez-Antilef ${ }^{a}$ (D), Camila Alejandra Heise-Barrera ${ }^{a}$ (D), Gloria Andrea Muñoz-Vidala (D), Genesis Estefanía Velásquez-Bórquez ${ }^{a}$ (D), Valeria Nicol Verdugo-Álvarez ${ }^{a}$ (D), Jimena Soledad Carrasco-Madariaga ${ }^{a}$ (D)

${ }^{a}$ Universidad Austral de Chile, Valdivia, Chile.

Cómo citar: Gómez-Antilef, B. F., Heise-Barrera, C. A., Muñoz-Vidal, G. A., Velásquez-Bórquez, G. E., Verdugo-Álvarez, V. N., \& Carrasco-Madariaga, J. S. (2020). Construcción de subjetividad trans a partir de la ocupación entendida como hacer, ser y llegar a ser. Cadernos Brasileiros de Terapia Ocupacional, 28(2), 419-435. https://doi.org/10.4322/2526-8910.ctoAO1960

\begin{abstract}
$\underline{\text { Resumen }}$
Introducción: Esta investigación indaga la influencia del sistema binario de género en la construcción del sujeto trans desde la perspectiva del Hacer y las potencialidades de Llegar a Ser, en el contexto sociocultural y político chileno, centrándose en las experiencias de los participantes mediante el rescate de la historia ocupacional. Objetivos: Analizar la construcción del sujeto trans a través del hacer, ser y llegar a ser en base a sus narrativas y en relación a la estructura social chilena en la cual se desenvuelven, visualizando la influencia en sus estados de salud. Metodo: Desde una perspectiva cualitativa con enfoque de conocimientos situados, se utiliza la metodología de producciones narrativas y técnicas discontinuas a múltiples voces. La información fue recolectada mediante entrevistas individuales y un grupo focal. Resultados: El proceso de tránsito se presenta como un continuo ocupacional, identificando la performatividad del género mediante la ocupación, así como las transformaciones corporales en la construcción de subjetividad. Además se identifica un contexto sociocultural y político que vulnera derechos, lo cual impacta en la salud y el involucramiento en ocupaciones colectivas. Conclusiones: La ocupación se presenta como un elemento reproductor del sistema binario de sexo-género, no obstante, también puede ser entendida como una herramienta de emancipación en la medida que permite transgredirla. En este sentido, se interpela a la Terapia Ocupacional a promover ocupaciones colectivas
\end{abstract}


que potencien la capacidad de agencia de sujetos abyectos, determinando estados de salud favorables.

Palabras-clave: Transgénero, Actividades Cotidianas, Performatividad de Género, Terapia Ocupacional.

\section{$\underline{\text { Resumo }}$}

Introduçáo: Esta pesquisa investiga a influência do sistema binário de gênero na construção do sujeito trans desde a perspectiva do fazer e as potencialidades do tornar-se a ser, no contexto sociocultural e político chileno, enfocando as experiências dos participantes através do resgate de História Ocupacional. Objetivos: Analisar a construção do sujeito trans através do fazer, ser e tornar-se, com base em suas narrativas e em relação à estrutura social chilena em que atuam, visualizando a influência em seus estados de saúde. Método: Em uma perspectiva qualitativa, com foco no conhecimento situado, foi utilizada a metodologia de produção de narrativa e técnicas descontínuas com múltiplas vozes. As informaçóes foram coletadas por meio de entrevistas individuais e um grupo focal. Resultados: $\mathrm{O}$ processo de trânsito é apresentado como um continuum ocupacional, identificando a performatividade de gênero através da ocupação, bem como transformaçóes corporais na construçáo da subjetividade. Além disso, é identificado um contexto sociocultural e político que viola os direitos, com impacto na saúde e no envolvimento em ocupações coletivas. Conclusóes: A ocupação é apresentada como um elemento reprodutor do sistema binário sexo-gênero, no entanto, também pode ser entendida como uma ferramenta de emancipação, na medida em que permite transgredi-la. Nesse sentido, interpela-se a Terapia Ocupacional a promover ocupaçóes coletivas que aumentem a capacidade de agência de sujeitos abjetos, determinando estados de saúde favoráveis.

Palavras-chave: Transgêneros, Atividades Cotidianas, Performatividade de Gênero, Terapia Ocupacional.

\section{$\underline{\text { Abstract }}$}

Introduction: This research inquires the influence of the binary gender system on the construction of the trans subject from the perspective of Doing and the potential of becoming, in the Chilean socio-cultural and political context, focusing on the experiences of the participants through the rescue of history occupational. Objectives: Analyze the construction of the trans subject through doing, being and becoming based on their narratives, the Chilean social structure in which they develop, visualizing the influence on their health states. Method: From a qualitative perspective with focus on situated knowledge, the methodology of narrative productions and discontinuous techniques is used to multiple voices. The information was collected through individual interviews and a focus group. Results: The transit process is presented as an occupational continuum, identifying the performativization of gender through occupation, such as bodily transformations, in the construction of subjectivity. In addition, a sociocultural and political context that violates rights is identified, which impacts on health and participation in collective occupations. Conclusions: Occupation is presented as a reproductive element of the binary sex-gender system, however, it can also be understood as a tool of emancipation insofar as it transgresses it. In this sense, Occupational Therapy 
is called upon to promote collective occupations that enhance the capacity of agency of abject subjects, determining favorable health states.

Keywords: Transgender, Activities of Daily Life, Gender Performativity, Occupational Therapy.

\section{Introducción}

En Chile, durante las décadas de 1980 y 1990 emergieron diversos movimientos sociales que tensionaron la relación entre el Estado y la población, siendo el movimiento "Lesbianas, Gays, Bisexuales y Transexuales" (en adelante LGBT) el que denunció la violencia que vivían estas personas y exigió derechos civiles (Garrido, 2016). En esos años, tanto la ley de penalización de la sodomía como de ofensas al pudor, moral y buenas costumbres, fueron utilizadas para criminalizar a hombres gays y personas trans (Garrido, 2016), lo cual se tradujo en violencia física, psicológica y/o sexual.

Con el inicio de la transición democrática, en 1991 se funda el Movimiento de Integración y Liberación Homosexual (en adelante MOVILH), primer paso hacia una articulación del movimiento LGBT en Chile. Sin embargo, al mismo tiempo, en los medios de comunicación proliferaron discursos que asociaban a esta población con la drogadicción, comercio y promiscuidad sexual. Esto, se puede entender como parte del efecto de inercia que, después del retorno a la democracia, mantuvo por muchos años el modelo de Estado impuesto por la dictadura. Dicho modelo, patriarcal y heterosexual, articuló los discursos estatales con los eclesiásticos de modo que, se siguió justificando la impunidad de quienes ejercían violencia hacia la comunidad LGBT (Garrido, 2016).

Las distintas formas de violencia hacia las personas trans han sido tema de preocupación y discusión académica de la mano del desarrollo de las perspectivas de género que, en una primera instancia, propusieron una diferencia entre sexo y género, donde el primero correspondía a algo biológico, mientras que el segundo, a una construcción sociocultural (Gil, 2002). A partir de esta distinción, se propuso que las personas trans "[...] sienten y expresan una identidad de género distinta del sexo biológico de nacimiento y, por tanto, socialmente esperado [...]” (Pino \& Verbal, 2015, p. 156).

Luego, las teorías de género han planteado críticas a esta concepción por mantenerse dentro de un sistema binario que, al igual como hace la dicotomía entre lo masculino y lo femenino, separa los aspectos biológicos y socioculturales, sin terminar con el sistema binario "[...] de forma que a un cuerpo de mujer le corresponde un género femenino, mientras que a un cuerpo de hombre le corresponde necesariamente un género masculino [...]" (Gil, 2002, p. 33). Es desde esta lógica binaria que se puede sostener que en las personas trans, falla esta correspondencia.

En oposición, Butler (2007) propone que, tanto el sexo como el género están socialmente construidos bajo un sistema binario de relaciones de poder, que busca mantener géneros inteligibles. Este sistema se reproduce a partir de la interpelación, que sería el "[...] mecanismo por el cual los aparatos de dominación actúan sobre los individuos para convertirlos en sujetos de su propia estructura de poder [...]" (Córdoba, 2003 , p. 89). Es decir, somos interpelados a identificarnos y actuar de una determinada forma en función de nuestro sexo, con lo cual se genera la ilusión de que el resultado de 
dicha interpelación, es decir, el género, es algo independiente y previo a este proceso, lo cual repercute "[...] en la forma en que las vidas serán vividas, en el refuerzo de los estigmas y en la propia dimensión subjetiva sobre sí [...]” (Maximiano, 2016, p. 220).

Butler propone que los sujetos no constituyen su identidad a partir de algo a priori, sino que, a partir de las formas concretas de estar en el mundo, las que, a su vez, construyen y reconstruyen los sistemas de poder hegemónicos existentes e influyen en la configuración de la experiencia de cada persona. Así, en vez de pensar en una identidad como una esencia previa, la autora propone pensar en una subjetividad que se produce por las experiencias y más específicamente, por medio de las transgresiones y/o negociaciones constantes con las macroestructuras.

El sujeto se construye y expresa a partir de la repetición constante de actuaciones en el mundo que contienen, por un lado, lo que el sistema de dominación interpela a hacer y por otro, las modificaciones que cada individuo introduce a la norma. Esto, porque cada vez que un acto es repetido se produce un desplazamiento, ya que una repetición nunca será idéntica a su referencia. Estas repeticiones constantes son lo que Butler (2007) llama actos performativos, que son también lo que construye y mantiene al género, permitiendo su reproducción y naturalización binaria (Gil, 2002). Así, las categorías subordinan y producen sujetos, pero al mismo tiempo, nos dan la posibilidad de acción en el mundo, que es justamente, lo que nos ofrecen la posibilidad de transgredirlas. En este proceso, el cuerpo se convierte en el elemento principal (Vartabedian, 2007), ya que es el receptor y el actor de cada experiencia.

La idea de que nos constituimos por medio de lo que hacemos o, en palabras de Butler (2007), por nuestras actuaciones en el mundo, es central para el concepto de ocupación. En ese sentido, podemos decir que el acto performativo se puede relacionar con la propuesta de Wilcock (1999) sobre el Ser, Hacer y Llegar a ser, según la cual, por medio de la ocupación, nos vamos transformando en un proceso continuo de crecimiento, transformación y auto-actualización, de manera que, finalmente, en cada momento somos el resultado de las cosas que hacemos. Así, la autora nos propone que existe un continuo entre el Ser, el Hacer y el Llegar a ser que se relaciona con "[...] las cosas que las personas hacen, la relación entre lo que hacen y lo que son como seres humanos y que está en estado constante de llegar a ser diferentes a través de la ocupación" (Wilcock, 1999, p. 3). Sin embargo, a diferencia de la idea de performatividad de Butler la propuesta de Wilcock presupone un Ser, es decir, una esencia previa a nuestras actuaciones (u ocupaciones) que se relaciona con el cuerpo biológico, como soporte material desde donde existimo y actuamos en el mundo. En tal sentido, el Ser que propone Wilcock no considera que lo que hacemos y, por lo tanto, lo que somos es, en parte, el resultado de lo que somos llamados, o interpelados a hacer, es decir, no considera el rol que tienen los aparatos de dominación en lo que hacemos ni en lo que somos.

Para efectos de esta investigación, el concepto de Ser (Wilcock, 1999) será reemplazado por la noción de subjetividad y, por lo tanto, no se asume una esencia previa a las actuaciones del género, sino que se entiende que el sujeto es llamado a identificarse socialmente con una identidad determinada. Sin embargo, nos interesa resaltar que, en este proceso, la ocupación juega un rol central, ya que implica las actuaciones de los sujetos en el mundo y, por lo tanto, contiene tanto las concepciones 
ideológicas de cada cultura y época (Le Breton, 1999 citado en González, 2001) así como la posibilidad de transformación.

Pero recordemos que la teoría de Wilcock, así como gran parte del desarrollo teórico al interior de la terapia ocupacional y las Ciencias de la Ocupación, se han centrado, más que en la noción de sujeto, en el concepto de salud, el cual se define como "[...] un equilibrio entre el bienestar físico, mental y social [...] alcanzado a través de ocupaciones significativas individuales y socialmente valoradas [...]" (Sanz \& Rubio, 2011 , p. 3), que para ser alcanzado, requiere que personas y grupos seamos capaces de "[...] identificar y desarrollar aspiraciones, satisfacer necesidades y cambiar o hacer frente a su entorno [...]” (Organización Mundial de La Salud, 1986, s.p.). Esta noción, es coherente con la idea de un Ser que se transforma por medio de la ocupación: de este modo, el equilibrio y el bienestar se alcanzarían por medio de ocupaciones que nos permitan mantenernos y/o transformarnos como Ser. Sin embargo, esta noción de salud no considera la influencia de las macroestructuras en la conformación del Ser que presuponen, ni en su transformación y/o mantención.

Por lo antes mencionado se hace necesario introducir un concepto de salud que nos permita articular las ideas de Wilcock con el tema de la subjetividad. En este sentido, Chapela \& Cerda (2011) destacan la reapropiación y agenciamiento colectivo para construir nuevas formas de subjetividad como la vía para una Promoción de Salud Emancipatoria. De este modo, un sujeto saludable sería aquél que puede acceder al campo político, además de agenciar soluciones a problemas individuales y colectivos.

En los últimos años la Terapia Ocupacional se ha preocupado de las personas trans, así como de otros grupos marginados. Acá, ha cobrado relevancia la realidad social, cultural y política. En específico, en el caso de las personas trans, se ha propuesto la desnaturalización de la norma binaria como guión de vida que dice quiénes son y cómo deben ser los sujetos (Maximiano, 2016). En vez de eso, deberíamos centrarnos en el hacer y las significaciones que encuentran estas personas en lo cotidiano; visualizando cómo las vivencias exigen una deconstrucción constante sobre cómo los procesos normativos inciden en las formas de vivir (Maximiano, 2016). En otras palabras, en las personas trans, más que pensar en una discordancia entre sexo y género, habría que pensar en cuerpos que se escapan de las categorías heteronormativas y de la norma binaria, y que por ello se encuentran en el lugar de la abyección, que es el espacio de la "no aceptación", por amenazar los patrones establecidos (Maximiano, 2016). Sin embargo, la abyección también construye al sujeto en cuanto lugar desde donde experimenta y actúa en el mundo. La pregunta que cabe entonces es qué rol juega la ocupación en este proceso. Esta pregunta cobra mayor sentido a la luz del estudio del Índice Compuesto de Estigma y Discriminación (ICED) aplicado a personas trans en Chile que muestra que, si bien la legislación chilena garantiza sus derechos civiles, aún quedan pendientes sus derechos económicos, sociales y culturales; se evidencian altos porcentajes de discriminación y exclusión hacia personas trans en contextos laborales, escolares y comunitarios (Programa Nacional de Prevención y Control del VIH/SIDA, 2012).

La presente investigación, tiene por propósito explorar qué papel tienen las ocupaciones en la reproducción de formas de exclusión y/o en el bien estar de las personas trans en el actual contexto chileno. Para ello, se realiza una reconstrucción de las historias ocupacionales de sujetos trans. Se asume que, en el involucramiento en ocupaciones, los seres humanos se van transformando y actualizando (Wilcock, 1999), de manera que, la ocupación es un aspecto constitutivo del sujeto. Al mismo tiempo, 
tomando aportes de las teorías de género y de la salud colectiva, se entiende que el contexto social y político también impactan en la subjetividad y, por ende, en el estado de salud y bien estar de personas y grupos. De este modo, junto con las historias ocupacionales, se busca analizar el actual contexto social y político chileno y como este afecta el involucramiento en ocupaciones y el estado de salud y bien estar.

\section{Metodología}

Esta investigación se posiciona en la perspectiva de los conocimientos situados (Haraway, 1995) y la investigación crítica (Montenegro et al., 2015). A través del diálogo entre investigadoras-participantes sobre diferentes temas, se generan una co-construcción de los conocimientos. La investigación responde a la metodología de producciones narrativas (Martinez \& Montenegro, 2010): el discurso de los participantes se considera un conocimiento concebido desde las propias experiencias de vida que posee el mismo valor que el conocimiento teórico de los investigadores. De este modo, se busca articular los saberes de ambos. Para ello es necesario que los participantes sepan cuales son los supuestos teóricos de los investigadores, de la misma forma como los investigadores buscan conocer las experiencias de vida de los participantes. La técnica utilizada corresponde a narrativas discontinuas a múltiples voces (Biglia \& Bonet-Martí, 2009) la cual consiste en confeccionar un documento que rescate la autonomía e individualidad del relato de cada participante y del investigador, existiendo el espacio para plantear diferentes posturas al mismo tiempo que se buscan los puntos de encuentro. De este modo se busca rescatar los puntos de vista del participante y del investigador.

Los criterios para la selección de los participantes fueron:

- Autodefinirse como persona trans;

- Ser mayor de 18 años;

- Tener interés de participar en alguna de las actividades de la investigación;

- Residir en la Región de Los Ríos o Los Lagos ${ }^{1}$.

La invitación a participar se realizó mediante Organizaciones LGTB de la ciudad de Valdivia, redes sociales y redes profesionales. De esta manera, se logró reunir a 6 voluntarios a quienes, se le dio la opción de participar en las actividades que ellos quisieran y/o tuvieran disponibilidad. Las actividades consideradas en la investigación en las cuales podían participar fueron 1) Confección de narrativas y 2) Grupos focales. Los datos fueron producidos entre los meses de mayo y octubre del año 2018.

Así, previa firma de Consentimiento Informado 6 voluntarios accedieron a participar.

\footnotetext{
${ }^{1}$ Las regiones son unidades territoriales y administrativas de la República de Chile. La Región de los Ríos tiene una superficie de 18.429,5 km cuadrados y una población de 384.837 habitantes según el censo 2017. Su capital es la ciudad de Valdivia, la que se ubica aproximadamente en los $39^{\circ}$ de latitud sur y $73^{\circ}$ de longitud oeste. La Región de los Lagos tiene una superficie de $48.584 \mathrm{~km}$ cuadrados y una población proyectada al 2015 de 828.708 habitantes según el censo 2017. Su capital es la ciudad de Puerto Mont, la que se ubica aproximadamente en los $41^{\circ}$ de latitud sur y $72^{\circ}$ de longitud oeste.
} 
Para la confección de narrativas, a cada participante se le entregó material resumido y de fácil comprensión, elaborado por las investigadoras respecto de los siguientes conceptos teóricos: Performatividad del género (Butler, 2007); Ser, Hacer y Llegar a ser (Wilcock, 1999) y Salud Emancipadora (Chapela \& Cerda, 2011). Se les pidió leerlo con el objetivo de que luego, pudieran dar su opinión con base en sus experiencias de vida respecto de dos temas: a) ocupación e identidad y, b) salud y ocupación. Luego, se realizaron dos sesiones de entrevista con cada participante, una por cada tema. Las entrevistas fueron grabadas, transcritas y utilizadas para elaborar un documento resumen donde se organizó la postura de cada entrevistado y del entrevistador. Dicho documento fue luego revisado y aprobado por cada uno de los participantes.

Luego, en el grupo focal se expuso a los participantes un breve resumen de los conceptos teóricos de la investigación y luego se les pidió reflexionar respecto de sus experiencias de vida. Finalmente, se analizaron documentos oficiales de las políticas chilenas sobre identidad de género y cambio corporal, los que fueron seleccionados por haber sido mencionados en las narrativas:

- Ley 21.120 Reconoce y da protección al derecho a la identidad de género (Chile, 2018);

- Circular 21 del Ministerio de Salud. Reitera instrucción sobre la atención de personas trans (Chile, 2012);

- Guía Clínica para la adecuación corporal en personas con incongruencia entre sexo físico e identidad de género, Ministerios de Salud (Chile, 2010).

La sesión tubo una duración de una hora y 45 minutos y fue facilitada por dos de las investigadoras. Fue grabada y luego transcrita.

En la siguiente Tabla 1 se presenta una caracterización de cada uno de los participantes, así como de las actividades de las que participaron:

Tabla 1. Caracterización, actividades en las que participan y fechas de generación de datos por participante.

\begin{tabular}{|c|c|c|c|c|c|c|}
\hline Identificación & Edad & $\begin{array}{l}\text { Lugar de } \\
\text { procedencia }\end{array}$ & $\begin{array}{l}\text { Lugar de } \\
\text { residencia }\end{array}$ & $\begin{array}{c}\text { Nivel } \\
\text { educacional }\end{array}$ & $\begin{array}{c}\text { Actividades } \\
\text { en las que } \\
\text { participa } \\
\end{array}$ & $\begin{array}{c}\begin{array}{c}\text { Fechas de } \\
\text { producción } \\
\text { de datos }\end{array} \\
\end{array}$ \\
\hline A & $\begin{array}{c}21 \\
\text { años }\end{array}$ & $\begin{array}{c}\text { Chiloé (Región } \\
\text { de Los Lagos) }\end{array}$ & $\begin{array}{c}\text { Osorno } \\
\text { (Región de } \\
\text { Los Lagos) }\end{array}$ & $\begin{array}{l}\text { Estudiante } \\
\text { universitario }\end{array}$ & $\begin{array}{l}\text { Producción } \\
\text { de narrativa y } \\
\text { Grupo Focal }\end{array}$ & $\begin{array}{c}\text { 1a Entrevista: }^{\text {19-08-18 }} \\
\text { 17-0 Entrevista: } \\
\text { 13-09-18 } \\
\text { Grupo Focal: } \\
\text { 13-10-08 }\end{array}$ \\
\hline An & $\begin{array}{c}24 \\
\text { años }\end{array}$ & $\begin{array}{c}\text { Valdivia } \\
\text { (Región de Los } \\
\text { Ríos) }\end{array}$ & $\begin{array}{l}\text { Valdivia } \\
\text { (Región de } \\
\text { Los Ríos) }\end{array}$ & $\begin{array}{l}\text { Estudiante } \\
\text { universitario }\end{array}$ & $\begin{array}{l}\text { Producción } \\
\text { de narrativa }\end{array}$ & $\begin{array}{c}\text { 1a Entrevista: }^{\text {a }} \\
\text { 26-05-18 } \\
\text { 2a Entrevista: } \\
\text { 21-06-18 }\end{array}$ \\
\hline C & $\begin{array}{c}19 \\
\text { años }\end{array}$ & $\begin{array}{c}\text { Río Bueno } \\
\text { (Región de Los } \\
\text { Ríos) }\end{array}$ & $\begin{array}{l}\text { Río Bueno } \\
\text { (Región de } \\
\text { Los Ríos) }\end{array}$ & $\begin{array}{l}\text { Estudiante } \\
\text { universitario }\end{array}$ & $\begin{array}{l}\text { Producción } \\
\text { de narrativa }\end{array}$ & $\begin{array}{c}\text { 1a Entrevista: }^{\text {a }} \text { 15-06-18 } \\
\text { 2a Entrevista: } \\
\text { 21-07-18 }\end{array}$ \\
\hline
\end{tabular}


Tabla 1. Continuación...

\begin{tabular}{|c|c|c|c|c|c|c|}
\hline Identificación & Edad & $\begin{array}{c}\text { Lugar de } \\
\text { procedencia }\end{array}$ & $\begin{array}{l}\text { Lugar de } \\
\text { residencia }\end{array}$ & $\begin{array}{c}\text { Nivel } \\
\text { educacional }\end{array}$ & $\begin{array}{c}\text { Actividades } \\
\text { en las que } \\
\text { participa }\end{array}$ & $\begin{array}{c}\text { Fechas de } \\
\text { producción } \\
\text { de datos }\end{array}$ \\
\hline $\mathrm{F}$ & $\begin{array}{c}32 \\
\text { años }\end{array}$ & $\begin{array}{c}\text { Santiago } \\
\text { (Región } \\
\text { Metropolitana) }\end{array}$ & $\begin{array}{l}\text { Valdivia } \\
\text { (Región de } \\
\text { Los Ríos). }\end{array}$ & Profesional & Grupo Focal & $\begin{array}{c}\text { Grupo Focal: } \\
13-10-08\end{array}$ \\
\hline $\mathrm{K}$ & $\begin{array}{c}21 \\
\text { años }\end{array}$ & $\begin{array}{l}\text { Temuco } \\
\text { (Región de la } \\
\text { Araucanía) }\end{array}$ & $\begin{array}{c}\text { Niebla } \\
\text { (Región de } \\
\text { Los Ríos) }\end{array}$ & $\begin{array}{c}\text { Estudiante } \\
\text { universitario }\end{array}$ & $\begin{array}{l}\text { Producción } \\
\text { de narrativa y } \\
\text { Grupo Focal }\end{array}$ & $\begin{array}{c}\text { 1a Entrevista: }^{\text {30-07-18 }} \\
\text { 2a Entrevista: } \\
\text { 23-08-18 } \\
\text { Grupo Focal: } \\
13-10-08\end{array}$ \\
\hline $\mathrm{T}$ & $\begin{array}{c}19 \\
\text { años }\end{array}$ & $\begin{array}{c}\text { Valdivia } \\
\text { (Región de Los } \\
\text { Ríos) }\end{array}$ & $\begin{array}{l}\text { Valdivia } \\
\text { (Región de } \\
\text { Los Ríos) }\end{array}$ & $\begin{array}{c}\text { Estudiante } \\
\text { universitario }\end{array}$ & Grupo Focal & $\begin{array}{c}\text { Grupo Focal: } \\
13-10-08\end{array}$ \\
\hline
\end{tabular}

Luego de un primer análisis de contenido de las narrativas y del grupo focal se definieron tres categorías para un análisis más detallado de los datos. Estas se definieron con base en los siguientes criterios: a) que estuvieran presentes en todas las narrativas y en el grupo focal y b) que fueran relevantes para responder a la pregunta de investigación. Así, la información se analizó de acuerdo con las siguientes categorías

1. Autopercepción del sexo/género: Influencias del contexto y de la ocupación;

2. TRANSitar: inicio, proyección y rol de la ocupación en el proceso;

3. Ser trans en el contexto sociopolítico chileno y avanzar hacia una salud emancipadora.

\section{Resultados}

\subsection{Autopercepción del sexo/género: Influencias del contexto y de la ocupación}

Tanto en las entrevistas como en el grupo focal, los participantes coinciden con que existe un binarismo que divide lo masculino de lo femenino asociado al sexo biológico y que impacta la vida cotidiana, por cuanto se genera una expectativa respecto de las características que los sujetos deben tener y los roles que deben cumplir. La existencia de este binarismo es atribuida a distintas causas, entre las cuales los participantes destacan la influencia de la Iglesia en la sociedad chilena, que impone una sexualidad con fines exclusivamente reproductivos, además de influir en que la familia replique esta norma.

Respecto de la forma en que los participantes perciben actualmente su propia identidad de género y su orientación sexual vemos que hay diferencias, aún cuando hay una tendencia a mantener una lógica binaria. Así, por ejemplo, $A$ y $A n$ se identifican con la figura masculina, y se consideran heterosexuales. Por su parte, $F$ se identifica como hombre heterosexual, mientras que $C$ también se identifica con la figura masculina, pero se considera homosexual. Por último, $K$ se distancia de esta tendencia y se identifica 
como trans, lo cual define como que su género "transita entre la dicotomía binaria” y como pansexual, es decir, que su atracción sexual hacia otro está dada por la esencia de este y no por su sexo o género.

Esta auto percepción se ha modificado en el tiempo. La mayoría reconoce un momento de ruptura que, como veremos más adelante, coincide con el inicio del proceso de transito y la redefinición tanto de la identidad de género, como de la orientación sexual. Así, $A, A n$ y $C$ mencionan haberse identificado antes como mujeres lesbianas, en especial durante su adolescencia.

Respecto del contexto social y cultural chileno, los participantes visualizan situaciones que hacen pensar que la población está más receptiva y tolerante respecto al tema trans. $K$ refiere que en su generación "[...] estaba más instaurado el tema de la Iglesia [...] existía mucha homofobia y transfobia”. F también cree que la violencia que existe actualmente hacia las personas trans más jóvenes es menor en comparación a periodos previos. Un ejemplo de ello sería lo ocurrido con el premio Oscar que ganó la película "Una Mujer Fantástica”, cuya protagonista es una mujer trans interpretada también por una actriz trans. Esto se considera algo positivo, pues visibilizó la temática y promovió el debate en torno a la Ley de Identidad de Género.

Pese a ello, se menciona que se mantiene un discurso machista y homofóbico que encasilla lo masculino en la norma binaria y desvaloriza la diversidad de expresiones del género incluso en los trans masculinos más jóvenes. Esto se ve reflejado en distintas situaciones de violencia simbólica que se siguen dando en círculos cercanos de los participantes, tales como malos tratos y discriminación en el contexto educativo, asociado al uso del nombre legal y discriminación en contextos laborales. Respecto de esto último, las situaciones que se mencionan no tienen relación con el hecho de ser trans sino que con tener actitudes que se vinculan a lo femenino. Un participante menciona, por ejemplo: "yo peinaba a mi compañera $y$, mis compañeros [...] me webiaban ${ }^{2}$ [...] esa confrontación igual me molestaba”.

Sin bien se identifica la importancia del contexto social y cultural, de las entrevistas se desprende que este no tiene el mismo impacto para todos: Los entornos más próximos, tales como la familia y el grupo de pares que describen los participantes dan cuenta de diferencias en la forma en que cada uno relata sus experiencias. Por ejemplo, $K$ y $C$ identifican expresiones discriminatorias en la escuela, en especial provenientes de sus pares, mientras que $A$ y $A n$ reconocen haber crecido en ambientes favorables, teniendo la oportunidad de expresar intereses y diversas formas de ser en el mundo.

Respecto de la ocupación, todos los participantes la visualizan como parte importante de la identificación con un género determinado y que, por lo tanto, ha sido fundamental en su proceso de tránsito. Varios reconocen que, previo a iniciar la transición, intentaron desarrollar ocupaciones socialmente concordantes con el género femenino lo cual interpretan ahora como un intento de alterar forzosamente su repertorio de intereses para adecuarse a la norma de género en concordancia con el sexo asignado al nacer. Al mismo tiempo, varios participantes mencionan haber desarrollado o haberse interesado por ocupaciones masculinas durante su infancia.

Así también, la ocupación habría sido importante en el proceso de identificación con el género masculino una vez iniciado el tránsito. Por ejemplo, $F$ refiere: "intenté ver

${ }^{2}$ Chilenismo. En el contexto de la frase se entiende como sinónimo de molestar, perturbar, ridiculizar. 
partidos de fútbol para sentirme más macho [...] quería ser aceptado y validado para poder aceptarme y validarme yo mismo". Al mismo tiempo, algunos participantes mantienen intereses o gustos asociados al género femenino luego de iniciado el tránsito. Por ejemplo, F menciona "me gusta tejer porque mi abuela cuando chico me enseñó, [además] me encanta peinar". Sin embargo, reconoce que esto no es bien visto por otros. También se mencionan las actividades de autocuidado como algo central en el proceso; $C$ y $A$ señalan la relevancia de las actividades de vestuario y arreglo personal para modificar su apariencia física y hacerla más masculina.

Durante el grupo focal, se plantea a los participantes discutir si la identidad de género es producida por un determinado contexto o si más bien, responde a una esencia que luego se expresa. Frente a ello F, plantea:

Es dificil saberlo [...] siento que desde chico sentí que no era mi cuerpo, ahora no tanto. Y no creía, y aún no creo tanto, que sea impuesto por otros [...] Yo senti eso, que habia nacido biológicamente [...], ahora también me lo cuestiono un poco, entonces mi respuesta sería no lo sé (Extracto grupo focal).

Por otra parte, $K$ señala:

Siento que siempre fui asi, [...] se dice que uno no tiene la edad como para definirse como trans [...], pero personalmente siempre he experimentado ciertos patrones masculinos de cuando era niño. $Y$ no sé, siento que siempre estuvo en realidad (Extracto grupo focal).

Al preguntar si esta vivencia puede tener que ver con el contexto social y familiar, A plantea:

En lo personal, mis papás siempre me dieron la libertad de hacer y ser quién yo quisiera. Entonces, nunca me vi como hombre o como mujer, bueno, siempre me identifiqué más como niño y a veces no entendía por qué otras personas me trataban como niña, era muy extraño (Extracto grupo focal).

Finalmente, todos los participantes identifican haber experimentado su identidad de género como una esencia que estaba oculta o reprimida. Más explícitamente, en las narrativas de $C$ y $A n$ se menciona la experiencia de haber nacido en un cuerpo equivocado. Estas experiencias parecen ser independientes del contexto social más próximo. Por otro lado, la ocupación habría jugado un rol fundamental en la identidad de género, ya sea para adecuarse a la norma binaria sexo/género o para sentirse más cercano a la propia identidad de género.

\subsection{TRANSitar: inicio, proyección y rol de la ocupación en el proceso}

El proceso de tránsito se inicia con un quiebre en la historia de vida de los participantes, que coincide con el reconocimiento de una identidad de género distinta a la femenina, que habría estado escondida y/o reprimida. En varios relatos este quiebre se asocia al encuentro con la temática trans. 
$A$ inicia su tránsito hace 7 meses, luego de "conocer lo trans" mediante una ONG que trabaja en el tema. Por su parte, An refiere que "accedió a lo trans" a partir de una película, siendo adulto-joven, lo que le ayudó a encontrar una coherencia entre su género y deseo. Previo a este momento, menciona haber estado en una disyuntiva que ahora atribuye a concepciones religiosas.

El proceso de tránsito es entendido por los participantes como una transformación que implica modificaciones en las formas cotidianas de hacer las cosas. Todos reconocen cambios en sus actividades de vestuario y arreglo personal que habrían sido centrales en este proceso pues les habrían permitido acercarse a la imagen que buscan proyectar.

Luego de las modificaciones en vestuario y arreglo personal, los participantes visualizan someterse a procedimientos médicos de cambio corporal como otro paso en el tránsito. Así, $A$ se encuentra decidido a iniciar tratamiento hormonal (en adelante hormonación) y mastectomía, mientras que $C$ ya ha iniciado los trámites necesarios para acceder a hormonación. Por su parte, $A n, K y F$ ya han iniciado hormonación, y refieren haber experimentando cambios físicos y emocionales, y mayor comodidad y confianza en sí mismos. Además de la hormonación, $K$ se ha sometido a mastectomía e histerectomía, y $F$ a adecuaciones corporales y cambio genital.

Todos quienes ya iniciaron procedimientos médicos para lograr un cambio corporal refieren sentirse más cómodos con su cuerpo y, además, manifiestan que esto ha implicado nuevos cambios en sus ocupaciones, tales como modificar actividades de autocuidado, en especial de higiene, e incorporar la administración de medicamentos en sus rutinas.

Todos los participantes refieren sentirse aún en este proceso, aún cuando se visualizan diversas perspectivas respecto de la proyección hacia el futuro. $A n, A$ y $C$ identifican el transito como un periodo acotado que, así como tubo un inicio, tendrá un fin. Por ejemplo, An refiere: "de aqui a que tenga un hijo voy a ser súper hombre". Por su parte, $C$ menciona: "va a ocurrir en un periodo de tu vida, [...] pero yo no pienso acarrear ese adjetivo por siempre, [...] hombre trans". En contraposición, $K$ y $F$ plantean que el transitar no tiene un punto de finalización, aún que reconocen que al inicio si creían que el proceso tendría término. $K$ pensaba "voy a sacarme los pechos, voy a tener testo y listo" y luego refiere "al final nunca dejé de ser trans para la gente". Por su parte $F$, quien inició su tránsito hace 15 años, refiere: "Antes pensaba que era un periodo hasta sentirme adecuado 100\%, ahora siento que quizás nunca va a terminar". Al preguntarles cómo proyectan el proceso hacia el futuro, $K$ nos dice que actualmente aspira a ser un trans queer, mientras que $F$ nos dice "[...] no sé a qué más podría llegar, pensé que habia llegado al tope [...] en cuanto a lo físico, porque en cuanto al rol creo que ya no hay más".

El cuerpo cobra un rol fundamental en el proceso de tránsito, ya sea como objeto de arreglo y cuidado y/o como algo a ser transformado por acción médica. Al respecto, los participantes manifiestan que, a medida que el proceso de tránsito avanza se van sintiendo cada vez más cómodos con sus cuerpos y que, por el contrario, antes o durante el primer periodo del tránsito se experimenta una incomodidad y problemas de autoestima. Así, por ejemplo, $K$ y $C$ se identifican actualmente como hombres atrapados en un cuerpo de mujer, a lo cual $C$ agrega que no se siente conforme con la ambigüedad de su cuerpo.

Durante el grupo focal, surgen cuestionamientos respecto de las motivaciones para el cambio corporal. Así, $F$ dice "ese sentirse más adecuado ¿será algo inconscientemente 
impuesto o no?” y $K$ agrega: “[...] a mi me pasó con los pechos. Dije: ¿me estoy sacando los pechos porque yo me siento incómodo [...] o porque la gente me hace sentir incómodo?”.

Finalmente, el proceso de tránsito se visualiza como algo que tiene un inicio, el cual suele coincidir con la adolescencia y con el acceso a información respecto de la transexualidad. Respecto de la proyección del proceso hay distintas posturas: algunos lo ven como algo que va a concluir en algún momento y otros como algo que nunca va a terminar. Todos los participantes dicen estar en este proceso, el cual caracterizan como un cambio en su apariencia física y manifiestan que, a medida que se avanza, hay mayores niveles de comodidad con el propio cuerpo. La ocupación, tiene un rol fundamental en el proceso, ya sea por la adopción de nuevas actividades de cuidado y arreglo personal y/o por la incorporación de nuevas rutinas en higiene y procedimientos médicos.

\subsection{Ser trans en el contexto sociopolítico chileno y avanzar hacia una salud emancipadora}

Durante el grupo focal se reflexiona sobre el rol que han tenido distintos organismos estatales en las vidas de los participantes y se concluye que existen dos instituciones que han vulnerado históricamente sus derechos: el poder judicial y el sistema sanitario. Esto, se ve expresado en los procedimientos que dichos órganos exigen cuando se solicita cambio de nombre y/o acceso a procesos médicos de transformación corporal. $F$ refiere:

Por orden del juez, aparte de tener el certificado del psiquiatra, te vuelven a hacer otra prueba psicológica en el médico legal, tienes que pasar por un examen físico, obligatorio, en muchos casos, te hacen empelotarte ${ }^{3}$ y te hacen sacar fotos. A mi, menos mal, me dijeron de antes que me negara a las fotos [...] y anotaron "la cavidad anal se ve intacta, por tanto, no ha habido penetración" (Extracto grupo focal).

Por su parte, Kagrega: "Te sentias como un bicho raro, como que el loco ponía una lupa con luz y anotaba. Pensaba cómo voy a pasar por toda esta vergüenza para que me validen". Contrario a esto, otro participante menciona estar de acuerdo con el procedimiento.

Se analizan declaraciones de diferentes actores políticos sobre el tema trans que dan cuanta de intolerancia y patologización, lo cual llevaría a que siempre se busque, en primer lugar, intentar sanar a quien manifiesta la intención de cambio de género. $\mathrm{Al}$ mismo tiempo, los participantes consideran que en estos discursos se confunden identidad de género y orientación sexual y cuestionan a quienes, lejanos a la experiencia trans, tienen el poder de aprobar los marcos legales reguladores de derechos.

Respecto de las normativas analizadas se concluye que, al no explicitar ni establecer los procedimientos, formas de fiscalización y de sanciones, no se logra resguardar los derechos de las personas trans.

Todos los participantes coinciden en que el contexto cultural y político incide en el estado de salud. Más concretamente, reconocen a la familia, organizaciones sociales, el saber médico y la Iglesia como estructuras sociales que han impactado en la construcción de subjetividad y en su estado de salud. $A n, K, A$ y $C$ cuentan que previo al inicio de su

${ }^{3}$ Chilenismo. En el contexto de la frase es sinónimo de “desnudarte”. 
transición fueron diagnosticados con patologías de salud mental, tales como trastornos alimenticios y del ánimo, asociadas a una supuesta disforia de género. Actualmente, si bien concuerdan con que tuvieron un problema de salud mental, ninguno cree que este se haya debido a su identidad de género. Por otra parte, todos los participantes concuerdan con que su estado de salud mejoró luego del inicio del proceso de transición.

Ninguno de los participantes sabía de la posibilidad de cambio de género en Chile y, la mayoría tubo su primer acercamiento a esta vía online y/o por organizaciones sociales, después de haber entrado en la adolescencia. En sintonía con lo anterior, todos han participado en acciones, colectivos y/o agrupaciones vinculadas al tema trans: $K$ menciona "[...] colectivamente trato de resolver dudas [de] cómo funciona el sistema de salud; tratar de agrupar a nivel de comunidad [...] pensando en la proyección. A mi me gusta caleta 4 el arte, entonces, he intentado de proyectar cuerpos trans"; $T$ menciona: "[...] fui activista muchos años [...] creo que cuando sali del closet, al tiro ${ }^{5}$ me meti a una agrupación y me entregaron tanto amor, tanta comprensión que siento que por eso mismo dejé de sentirme mal'. A también menciona haber compartido información con un chico trans de Valdivia y $F$ refiere "[...] no soy de hacerlo visible, pero lo intento hacer ayudando a los chicos más nuevos, registrando mis procesos, contándoles las cosas que me salieron mal y bien. También hice terapia a chicos trans gratis". Por su parte, An dice que fue importante ayudar a otros mediante el uso de redes sociales.

A pesar de realizar estas acciones los participantes no se visualizan a sí mismos como sujetos políticos de cambio, no obstante, durante el grupo focal, las investigadoras proponen que el rescate de las narrativas trans pueden ser una práctica emancipadora de Promoción de la Salud y los participantes reconocen esto como algo fundamental para promover estados de salud favorables.

La preocupación por la salud también es abordada respecto de los procesos de transformación corporal, sin embargo, en este caso no se habla de salud mental. Por ejemplo, $K$ menciona que su salud se ha visto afectada por su identidad de género, ya que "[...] si hubiera tenido mi cuerpo normal no tendría que preocuparme de cosas que otras personas no les preocupa", refiriéndose a las complicaciones de la menopausia luego de la histerectomía y al cuidado de las cicatrices luego de la mastectomía. Por su parte, $K$ menciona " $n$ o existen estudios cientificos sobre los efectos del tratamiento hormonal a largo plazo". Varios participantes imaginan situaciones catastróficas si, por alguna razón, no pueden acceder a tratamiento hormonal.

\section{Discusión}

A partir de las narrativas y el grupo focal, podemos concluir que los participantes identifican macroestructuras, tales como el marco jurídico, el sistema sanitario, el saber biomédico y la iglesia, entre otros, como partes de un sistema de dominación que ha impactado en sus historias de vida, imponiéndoles una identidad de género con la cual no se identifican, con base en el sexo asignado al nacer. Sin embargo, para los participantes su identidad de género no es el resultado de la constante interpelación que estas macroestructuras hacen. Por el contrario, sus vivencias apuntan más bien a que su

\footnotetext{
${ }^{4}$ Chilenismo: en el contexto de la frase es sinónimo de "mucho".

${ }^{5}$ Chilenismo. En el contexto de la frase es sinónimo de "inmediatamente".
} 
identidad de género era algo previo al inicio de su proceso de tránsito. Luego, en la medida en que inician su tránsito, van modificando sus actividades de la vida diaria, ya sea para dejar de lado actividades que consideran más propias del género femenino, involucrarse en ocupaciones que identifican como propias de su género, o para lograr una apariencia más acorde con este último. Los participantes identifican las actividades de vestuario, arreglo personal y autocuidado asociado a los procedimientos médicos de transformación corporal como fundamentales para un mejor estado de salud. De este modo, podemos concluir que el cambio ocupacional es parte fundamental del tránsito y que este, a su vez, es fundamental para la salud y bien estar.

Los participantes comprenden el proceso de tránsito como ir avanzando hacia una forma de ser cada vez más fiel a su identidad o esencia. Por medio de acciones buscan adecuar sus roles y su apariencia física a dicha identidad. En tal sentido, la vivencia de los participantes es concordante con la teoría de Wilcock (1999): Habría un Ser previo a las actuaciones de género. Dicho Ser (o identidad), por lo general, se corresponde con un género que se enmarca en la norma binaria pero que entra en conflicto por no ser concordante con el sexo asignado al nacer. Esto nos lleva a preguntarnos por aquello que es previo a nuestras actuaciones en el mundo o, más específicamente, por la dimensión material del Ser que nos propone Wilcock (1999). A diferencia de lo propuesto por Butler, esto nos permite pensar en el cuerpo biológico sin caer en determinismos como los que han negado la existencia de sujetos trans en el pasado, y entenderlo como el lugar a partir del cual estamos y actuamos en el mundo.

Retomando la propuesta de Maximiano (2016), si nos centramos en el hacer de los sujetos y las significaciones que estos encuentran en lo cotidiano podemos decir que la ocupación es parte constituyente del tránsito. A esto, podemos agregar que, estas ocupaciones también están influenciadas por la norma binaria de género.

Por otra parte, el cambio corporal, es una forma de reapropiación y agenciamiento que permite construir nuevas formas de subjetividad a los participantes y alcanzar mayores niveles de salud y bien estar (Chapela \& Cerda, 2011). Sin embargo, para acceder a este cambio los participantes deben regirse por protocolos que vulneran sus derechos, los cuales hacen parte de una concepción de la salud impuesta por el paradigma biomédico que los sitúa en el lugar de pacientes.

Este contexto social y político de vulneración de derechos de las personas trans, hace que los participantes busquen apoyo, y luego, intenten dar apoyo a otros/as personas trans, con lo cual, además de las ocupaciones propias de su tránsito, se involucran con otros y van generando redes de apoyo mutuo que son importantes en el proceso ya que generan mayor bienestar. Esto, en el proceso de la investigación es relevado como algo fundamental para promover mayores niveles de bienestar entre las personas trans y, por lo tanto, como parte de un proceso de Salud Emancipatorio, distinto del proceso de salud que se propone desde el modelo biomédico.

Las ocupaciones descritas por los participantes pueden interpretarse como un medio para lograr una adecuación a un género que se enmarca en la norma binaria (masculino / femenino). En este sentido, podemos decir que estas ocupaciones, contienen las concepciones ideológicas de nuestra cultura y época (Le Breton, 1999 citado en González, 2001). Estas se estructuran a partir de una forma socialmente aceptada, tal como lo han propuesto distintos autores con el concepto Forma Ocupacional (Nelson, 
1988; Kielöfner, 2002). Por lo tanto, hacen parte de un sistema de dominación que interpela a hacer las cosas de una cierta manera.

Sin embargo, en los sujetos trans, estas ocupaciones también transgreden la norma binaria por cuento implican una discordancia entre sexo y género. Por lo tanto, las ocupaciones de estos sujetos tienen el potencial de lo que Butler (2007) concibe como actos performativos ya que contienen la posibilidad de introducir modificaciones a las formas socialmente aceptadas o, en palabras de Nelson (1988), introducir algo nuevo cada vez que atraviesan una Forma Ocupacional, en este caso, algo propio de la subjetividad trans.

La patologización de la condición trans en el sistema de salud se puede entender como una respuesta a la transgresión que esta implica (Pérez, 2017) y, por tanto, hace parte de los sistemas que buscan perpetuar el orden establecido. En ese sentido, proponemos que la terapia ocupacional, como disciplina del área de la salud debe introducir un concepto distinto al que tradicionalmente se ha propuesto desde el modelo biomédico. Proponemos que, en el desarrollo teórico de la disciplina, sanar no debe ser un proceso anti-enfermedad al que alguien se somete, sino el producto de prácticas significantes (Chapela \& Cerda, 2011) relacionadas con ocupaciones individuales y colectivas que permitan a los individuos auto-sujetarse y ser agentes sociales con posibilidad de diseñar, decidir y lograr futuros viables, para así influenciar sus estados de salud. En el caso de las personas trans, esto requiere además cuestionar la separación mente-cuerpo, propia de la mirada biomédica tradicional, ya que mantiene la separación entre los aspectos biológicos y socioculturales a la base de la distinción entre sexo y género (Gil, 2002).

\section{Conclusión}

Retomando nuestros supuestos teóricos, que plantea que, el llamado a identificarse con una determinada identidad de género se sustenta sobre la base ilusoria, de una supuesta esencia que estuvo allí previo a la interpelación (Córdoba, 2003), creemos que es fundamental no desestimar los relatos de los participantes ni invalidar sus experiencias. En este sentido, creemos que la propuesta de Maximiano (2016) de desnaturalizar la norma binaria como guión de vida es pertinente sólo en la medida en que, pese al proceso de tránsito, los participantes siguen teniendo la vivencia de que sus cuerpos se encuentran en un lugar de "no aceptación". De este modo, en este trabajo hemos intentado invitar a los participantes a un diálogo que problematiza la idea de una identidad de género como una esencia que estaba oculta o reprimida y abrir la posibilidad de visualizar esta identidad como una construcción constante, no con el fin de imponer una teoría a sus vivencias sino, para abrir la posibilidad de comprender esta sensación de nunca poder llegar a ser del todo como se desea, como el lugar de la abyección que escapa a la norma binaria y que, por lo mismo es el lugar desde donde es posible amenazar los patrones establecidos.

Los resultados de esta investigación no pretende ser una representación posible de extrapolar a generalidades respecto de las personas trans. Además, no se incluyó a sujetos trans que se identificaran con el género femeninos, lo cual acota aún más los resultados.

Finalmente se concluye la necesidad de generar más investigación que se orienten a articular el conocimiento teórico de la terapia ocupacional con la vivencia de los sujetos 
trans. Para futuras investigaciones creemos que sería provechoso hacer una mayor indagación en los protocolos de procedimientos médicos de cambio corporal y sus implicancias en el cambio ocupacional de los sujetos.

\section{Referencias}

Biglia, B., \& Bonet-Martí, J. (2009). La construcción de narrativas como método de investigación psicosocial. Prácticas de escritura compartida. Forum Qualitative Social Research, 10(1), 1-26. Recuperado el 27 de marzo de 2018, de http://nbn-resolving.de/urn:nbn:de:0114-fqs090183

Butler, J. (2007). EI género en disputa: el feminismo y la subversión de la identidad. Barcelona: PAIDÓS.

Chapela, M., \& Cerda, A. (2011). Investigación cualitativa sanadora. In C. Martinez (Ed.), Por los caminos de la investigación cualitativa. Exploraciones narrativas y reflexiones en el ámbito de la salud (pp. 120-138). México: Editorial Series Académicos CBS.

Chile. (2018, 10 de diciembre) Ley 21.120. Reconoce y da protección al derecho a la identidad de género. Biblioteca del Congreso Nacional, Santiago.

Chile. Ministerio de Salud. (2010). Via Clinica para la adecuación corporal en personas con incongruencia entre sexo fisico e identidad de género. Chile: Ministerio de Salud.

Chile. Ministerio de Salud. (2012, 14 de junio). Reitera Instrucciones sobre la atención de personas trans em la red assistencial (Circular no 21). Santiago: Ministerio de Salud.

Córdoba, D. (2003). Identidad sexual y performatividad. Athenea Digital, 4, 87-96. Recuperado el 8 de marzo de 2017, de http://atheneadigital.net/article/view/n4-cordoba/87-pdf-es

Garrido, C. (2016) Historias de un pasado cercano. Memoria colectiva, discursos y violencia homolesbotransfóbica en la dictadura militar y transición democrática en Chile (Disertación de grado). Universidad Diego Portales, Chile.

Gil, E. P. R. (2002). ¿Por qué le llaman género cuando quieren decir sexo?: Una aproximación a la teoría de la performatividad de Judith Butler. Athenea Digital, (2), 30-41. Recuperado el 17 de marzo de 2017, de https://atheneadigital.net/article/view/n2-gil

González, A. (2001). Imagen corporal: cuerpo vivido, cuerpo escindido. Perinatologia y Reproduccion Humana, 15(2), 145-151.

Haraway, D. (1995). Ciencia, cyborgs y mujeres: la reinvención de la naturaleza. Valencia: Ediciones Cátedra.

Kielöfner, G. (2002). Model of human occupation. Philadelphia: Lippincott Williams \& Wilkins.

Martinez, A., \& Montenegro, M. (2010). Narrativas en torno al trastorno de identidad sexual: de la multiplicidad transgénero a la producción de trans-conocimientos. Prisma Social, 4(2), 1-44.

Maximiano, K. (2016). Terapia Ocupacional Social, pessoas trans e Teoria Queer: (re) pensando concepçóes normativas baseadas no gênero. Cadernos de Terapia Ocupacional da UFSCar, 24(1), 215-223.

Montenegro, M., Pujol, J., \& Vargas, L. (2015). Miradas, formas de hacer y relaciones en la constitución de una investigación crítica. Universitas Psychologica, 14(5), 1833-1852.

Nelson, D. (1988). Occupation: form and performance. The American Journal of Occupational Therapy, 42(10), 633-641.

Organización Mundial de La Salud - OMS. (1986). Carta para la promoción de la salud. Ottawa: Asociación Canadiense de Salud Pública.

Pérez, P. (2017). Rosa Parks con Judith Butler: performatividad individual y acción colectiva. Isegoría, (56), 187-204.

Pino, S., \& Verbal, V. (2015). La identidad de género en Chile, problematizando el debate. Revista de Psicología Universidad de Chile, 1(7), 155-178. 
Programa Nacional de Prevención y Control del VIH/SIDA., \& Ministerio de Salud. (2012). Índice Compuesto de Estigma y Discriminación hacia hombres homosexuales, otros HSH y mujeres transgénero en Chile (ICED): Sintesis de Estudio. Chile: Quad/Graphics. Recuperado el 19 de agosto 2018, de http://www.onu.cl/onu/wp-content/uploads/2013/01/ESTUDIO-ESTIGMA-YDISCRIMINACION-HSH-Y-MUJERES-TRANS.-CHILE-dic-2012.pdf

Sanz, P., \& Rubio, C. (2011). Eleanor Clarke Slagle. Fundadora y “madre” de la terapia ocupacional. Su legado. Revista Terapia Ocupacional Galicia, 8(13), 1-19. Recuperado de: http://www.revistatog.com/num13/pdfs/historia1.pdf

Vartabedian, J. (2007). El cuerpo como espejo de las construcciones de género. Una aproximación a la transexualidad femenina. Quaderns-e, 10, 1-14. Recuperado de: https://www.raco.cat/index.php/QuadernseICA/article/view/109038/136554

Wilcock, A. (1999). Reflections on doing, being and becoming. Australian Occupational Therapy Journal, $46,1-11$.

\section{Contribución de los Autores}

Bárbara Francisca Gómez-Antilef, Camila Alejandra Heise-Barrera, Gloria Andrea Muñoz-Vidal, Genesis Estefanía Velásquez-Bórquez y Valeria Nicol VerdugoÁlvarez: Planteamiento del tema y diseño, toma y análisis de datos, organización de resultados. Jimena Soledad Carrasco-Madariaga: Diseño metodológico, organización de resultados y redacción del texto. Todos los autores aproban la version final del texto.

\section{Fuentes de Financiamiento}

Proyecto FONDECYT 11160195.

\section{Autor para la correspondencia}

Jimena Soledad Carrasco-Madariaga

e-mail: jimenacarrasco@uach.cl 